\title{
Comparación de dimensiones oclusales en dentición decidua completa entre niños de 3 a 5 años de la Clínica Estomatológica Central de la Universidad Peruana Cayetano Heredia y el distrito de Yamango (Piura) en el año 2011
}

\section{Resumen}

Objetivo: determinar y comparar las dimensiones oclusales en dentición decidua completa entre niños residentes en el distrito de Yamango en el departamento de Piura con modelos de estudio de niños en dentición decidua completa atendidos en la Clínica Estomatológica Central en la ciudad Lima. Material y métodos: se evaluaron modelos de estudio de 124 niños, 56 hombres y 68 mujeres, de 3 a 5 años de edad con
Rubén Enrique- Zambrano- Maguiña ${ }^{1}$

Abraham-Meneses -López ${ }^{2}$

Fernando-Silva-Esteves -Raffo ${ }^{2}$

Artigo Original

\section{Comparação da dimensão oclusal da dentição decídua completa entre crianças de 2 a 5 anos da Clínica Estomatológica Central da Universidade Peruana Cayetano Heredia e o distrito de Yamango (Piura), no ano de 2011}

Resumo

Objetivo: Determinar e comparar as dimensões oclusais na dentição decídua completa entre oclusión normal y maloclusión clase I. Se utilizó la prueba de U de Mann- Whitney $(\mathrm{p}<0.05)$ para evaluar diferencias entre sujetos por lugar de procedencia, sexo y edad. Resultados: se reportaron medidas promedio, desviación estándar y significancia estadística. Se encontraron diferencias significativas en todas las dimensiones oclusales comparadas por procedencia, sexo y edad.

Palabras clave: Dimensiones oclusales, dentición decidua, maloclusiones. 
na cidade de Lima. Material e Método: foram avaliados modelos de estudo de 124 crianças, 56 masculinos e 68 femininos, de 3 a 5 anos de idade com oclusão normal e maloclusões classe I. Foi utilizado o teste de U de Mann-Whitney $(\mathrm{p}<0.05)$ para avaliar diferenças entre crianças por lugar de procedência, sexo e idade. Resul- tados: foram obtidas medidas médias, desvios padrões de significancia estatística, e foram encontrados diferenças significativas em todas as dimensões oclusais comparadas por procedência, sexo e idade.

Palavras Chaves: Dimensões oclusais; dentição decídua; maloclusões.

\section{Comparison of occlusal dimensions complete primary dentition among children aged 3 to 5 years of the Central Dental Clinic of Cayetano Heredia Peruvian University Central and Yamango (Piura) in 2011}

\begin{abstract}
The purpose of this study was to dertermine and compare the oclusal dimensions complete primary dentition in children living in the district of Yamango in the department of Piura to study models of children complete primary dentition seen in the Central Dental Clinic in the city Lima. Study models were assessed in 124 children, 56 men and 68 women, 3 to 5 years of age with normal occlusion and Class I malocclusion. We used the U Mann-Whitney test $(p<0.05)$ to assess differences between subjects by place of origin, sex and age. Measures were reported average, standard deviation and statistical significance. Significant differences were found in all dimensions compared to oclusal origin, sex and age.
\end{abstract}

Keywords: Dimensions occlusal, primary dentition, maloclussion

\section{Introducción}

La dentición decidua o primaria es la primera que el ser humano presenta en sus primeros años de vida, la cual se inicia desde el cuarto al sexto mes de vida intrauterina haciendo erupción en boca entre los seis meses hasta los treinta meses de edad post natal. Estos veinte dientes deciduos servirán de guía para la erupción adecuada de los dientes permanentes debido a que mantienen el espacio necesario para un posicionamiento adecuado en boca de los dientes sucedáneos.

Existen estudios sobre las dimensiones oclusales en dentición decidua completa para determinar un promedio de ellas, como las realizadas en un inicio por Baume ${ }^{1}$ y posteriormente por Moorrees $^{2}$ y Moyers ${ }^{3}$. Estas medidas se empezaron a tomar como referencia en estudios similares $^{4,5,6,8}$, para poder diagnosticar con anticipación ${ }^{7}$ e interceptar maloclusiones en dentición decidua. Estas dimensiones oclusales son: Ancho bicanino, Ancho bimolar, Longitud de Arco y Perímetro de Arco.

El motivo de este estudio tiene importancia social, debido a que el lugar de la investigación (Distrito de Yamango en el Departamento de 
Piura) no ha sido considerado anteriormente por un estudio similar al presente. Esto será beneficioso debido a que se podrá obtener información teórica acerca de las dimensiones oclusales en niños de 3 a 5 años en dentición decidua completa residentes del Distrito de Yamango y compararla con la muestra de la Clínica Estomatológica Central de la Universidad Peruana Cayetano Heredia y discutir sus semejanzas o diferencias.

\section{Material y métodos}

El diseño del estudio fue de tipo transversal, observacional y comparativo, sobre 144 modelos de estudio en dentición decidua completa de pacientes atendidos en la Clínica Estomatológica Central de la Universidad Peruana Cayetano Heredia y niños residentes en el distrito de Yamango en el Departamento de Piura en el año 2011, que cumplieron con los criterios de selección. Se evaluaron las dimensiones oclusales.

Se midieron los siguientes indicadores: (Figura A: 1, 2, 3).

- Ancho bicanino (Fig. A: 1): La distancia que existe entre la cúspide del canino deciduo derecho y la cúspide del canino deciduo izquierdo.

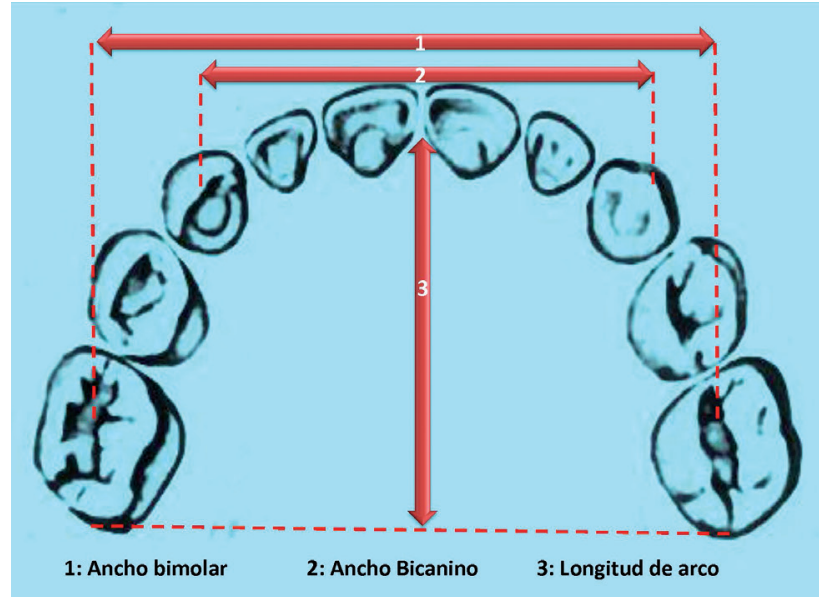

Figura A. 1, 2 y 3.

- Ancho bimolar (Fig. B: 2): La distancia que existe entre la fosa central de la segunda molar decidua derecha y la segunda molar decidua izquierda.

- Longitud de arco (Fig. A: 3): La distancia que existe entre la tangente a las caras distales de las segunda molares deciduas al punto más vestibular de incisivos, en su zona media.

Para el análisis de datos se utilizó la media aritmética, desviación estándar y prueba $U$ de Mann-Whitney para evaluar diferencias entre los grupos por lugar de procedencia, sexo y edad, mediante el programa estadístico SPSS v. 15.0.

Tabla 1. Comparación de dimensiones o clusales (sexo/pro cedencia)

\begin{tabular}{|c|c|c|c|c|c|c|c|c|c|c|}
\hline & \multicolumn{5}{|c|}{ Lima } & \multicolumn{5}{|c|}{ Yamango } \\
\hline & \multicolumn{2}{|c|}{ Hombre } & \multicolumn{2}{|c|}{ Mujer } & \multirow[b]{2}{*}{$\mathbf{P}$} & \multicolumn{2}{|c|}{ Hombre } & \multicolumn{2}{|c|}{ Mujer } & \multirow[b]{2}{*}{$\mathbf{P}$} \\
\hline & $x$ & D.S. & $\mathbf{x}$ & D.S. & & $x$ & D.S. & $x$ & D.S. & \\
\hline$A B C S$ & 31.7 & 1.97 & 30.8 & 1.64 & 0.09 & 29.8 & 2.42 & 28.8 & 1.93 & 0.05 \\
\hline $\mathrm{ABCl}$ & 25.4 & 2.32 & 24.9 & 2.77 & 0.00 & 24.5 & 2.13 & 22.7 & 1.86 & 0.47 \\
\hline ABMS & 41.1 & 1.96 & 40.8 & 2.16 & 0.00 & 40.5 & 2.18 & 38.9 & 2.2 & 0.65 \\
\hline ABMI & 35.9 & 1.74 & 34.9 & 2.99 & 0.47 & 34.4 & 3.55 & 33.8 & 2.88 & 0.14 \\
\hline LAS & 28.9 & 2.35 & 28.3 & 2.05 & 0.18 & 26.8 & 2.35 & 26 & 1.73 & 0.22 \\
\hline LAI & 25.9 & 1.68 & 25.7 & 2.76 & 0.58 & 23.6 & 2.22 & 23.3 & 1.65 & 0.76 \\
\hline
\end{tabular}




\section{Resultados}

El presente estudio describe el promedio de las dimensiones oclusales en dentición decidua completa de 124 niños, 56 hombres y 68 mujeres, de 3 a 5 años de edad, con oclusión normal y maloclusión clase I, dividido en dos grupos por lugar de procedencia: Lima y Yamango; por sexo: Hombres y Mujeres; por edades: 3, 4 y 5 años con 19, 77 y 28 sujetos respectivamente.

El presente estudio describe y compara los resultados de las dimensiones oclusales de los arcos dentarios deciduos de 144 niños (62 niños de Lima y 62 niños de Yamango) en tres grupos de edades 3,4 y 5 años.

\section{Comparación de dimensiones oclusales (sexo/procedencia)}

En la tabla 1, se observan los valores de las dimensiones oclusales en dentición decidua completa de los niños de Lima y Yamango, los valores promedio fueron:

Para los niños de Lima:

- Ancho bicanino superior $31.7 \mathrm{~mm}$, ancho bicanino inferior $25.4 \mathrm{~mm}$.

- Ancho bimolar superior $41.1 \mathrm{~mm}$, ancho bimolar inferior $35.9 \mathrm{~mm}$.

- Longitud de arco superior 28.9 mm y longitud de arco inferior $25.9 \mathrm{~mm}$.

Para las niñas de Lima:

- Ancho bicanino superior $30.8 \mathrm{~mm}$, ancho bicanino inferior $24.9 \mathrm{~mm}$.

- Ancho bimolar superior $40.8 \mathrm{~mm}$, ancho bimolar inferior $34.9 \mathrm{~mm}$.
- Longitud de arco superior $28.3 \mathrm{~mm}$ y longitud de arco inferior $25.7 \mathrm{~mm}$.

Al ser comparadas por sexo según procedencia, se obtuvo en el ancho bicanino inferior y ancho bimolar superior una diferencia estadística altamente significativa $(\mathrm{p}<0.01)$.

Los valores en el grupo Yamango fueron para niños:

- Ancho bicanino superior $29.8 \mathrm{~mm}$, ancho bicanino inferior $24.5 \mathrm{~mm}$.

- Ancho bimolar superior $40.5 \mathrm{~mm}$, ancho bimolar inferior $34.4 \mathrm{~mm}$.

- Longitud de arco superior $26.8 \mathrm{~mm}$ y longitud de arco inferior $23.6 \mathrm{~mm}$.

Para las niñas fueron:

- Ancho bicanino superior $28.8 \mathrm{~mm}$, ancho bicanino inferior $22.7 \mathrm{~mm}$.

- Ancho bimolar superior $38.9 \mathrm{~mm}$, ancho bimolar inferior $33.8 \mathrm{~mm}$.

- Longitud de arco superior $26.0 \mathrm{~mm}$ y longitud de arco inferior $23.3 \mathrm{~mm}$.

Al ser comparadas por sexo según procedencia, se obtuvo en el ancho bicanino superior una diferencia estadística significativa $(\mathrm{p}<0.05)$.

\section{Comparación de dimensiones oclusales (procedencia/sexo)}

En la tabla 2, se observan los valores de las dimensiones oclusales en dentición decidua completa (los mismos que en la tabla 1), pero esta vez se realizó la comparación.

Por procedencia según sexo, en niños: 
Tabla 2. Comparación de dimensiones o clusales (pro cedencia/sexo)

\begin{tabular}{lccccccccccc}
\hline \multicolumn{1}{c}{ Hombre } & \multicolumn{4}{c}{ Lima } & \multicolumn{3}{c}{ Yamango } \\
\hline & \multicolumn{4}{c}{ Lima } & \multicolumn{3}{c}{ Yamango } & \multicolumn{4}{c}{ Mujer } \\
& $\mathbf{X}$ & D.S. & $\mathbf{X}$ & D.S. & $\mathbf{P}$ & $\mathbf{X}$ & $\mathbf{D . S}$. & $\mathbf{X}$ & $\mathbf{D . S .}$ & $\mathbf{P}$ \\
ABCS & 31.7 & 1.97 & 29.8 & 2.42 & 0.54 & 30.8 & 1.64 & 28.8 & 1.93 & $\mathbf{0 . 0 0}$ \\
ABCI & 25.4 & 2.32 & 24.5 & 2.13 & 0.76 & 24.9 & 2.77 & 22.7 & 1.86 & $\mathbf{0 . 0 0}$ \\
ABMS & 41.1 & 1.96 & 40.5 & 2.18 & 0.99 & 40.8 & 2.16 & 38.9 & 2.20 & $\mathbf{0 . 0 1}$ \\
ABMI & 35.9 & 1.74 & 34.4 & 3.55 & 0.20 & 34.9 & 2.99 & 33.8 & 2.88 & 0.54 \\
LAS & 28.9 & 2.35 & 26.8 & 2.35 & $\mathbf{0 . 0 5}$ & 28.3 & 2.05 & 26.0 & 1.73 & $\mathbf{0 . 0 0}$ \\
LAI & 25.9 & 1.68 & 23.6 & 2.22 & $\mathbf{0 . 0 0}$ & 25.7 & 2.76 & 23.3 & 1.65 & $\mathbf{0 . 0 0}$ \\
\hline
\end{tabular}

$U$ de Mann Whitney / $p<0.05$

- Se obtuvo una diferencia estadística significativa en la longitud de arco superior $(\mathrm{p}<0.05)$ y una diferencia estadística altamente significativa en la longitud de arco inferior $(\mathrm{p}<0.01)$.

Por procedencia según sexo, en niñas:

- Se obtuvo una diferencia estadística altamente significativa en el ancho bicanino superior, ancho bicanino inferior, ancho bimolar superior, longitud de arco superior y longitud de arco inferior $(\mathrm{p}<0.01)$.

\section{Comparación de dimensiones oclusales (procedencia/ 3 años de edad)}

En la tabla 3, se obtuvieron los valores promedios de las dimensiones oclusales para niños de 3 años.

Para Lima fueron:

- Ancho bicanino superior $29.8 \mathrm{~mm}$, ancho bicanino inferior $23.9 \mathrm{~mm}$.

- Ancho bimolar superior $40.0 \mathrm{~mm}$, ancho bimolar inferior $34.9 \mathrm{~mm}$.
- Longitud de arco superior $25.9 \mathrm{~mm}$ y longitud de arco inferior $23.6 \mathrm{~mm}$.

Para Yamango fueron:

- Ancho bicanino superior $30.5 \mathrm{~mm}$, ancho bicanino inferior $24.7 \mathrm{~mm}$.

- Ancho bimolar superior $39.3 \mathrm{~mm}$, ancho bimolar inferior $35.5 \mathrm{~mm}$.

- Longitud de arco superior $28.5 \mathrm{~mm}$ y longitud de arco inferior $26.7 \mathrm{~mm}$.

Al comparar los valores de las dimensiones oclusales, se obtuvo una diferencia estadística altamente significativa en la longitud de arco inferior $(p<0.01)$.

Tabla 3. Comparación de dimensiones oclusales (procedencia / 3 años de edad).

\begin{tabular}{lccc}
\hline & \multicolumn{3}{c}{ 3 años } \\
& Yamango & Lima & P \\
\hline ABCS & 29.8 & 30.5 & 0.48 \\
ABCI & 23.9 & 24.7 & 0.54 \\
ABMS & 40.0 & 39.3 & 0.42 \\
ABMI & 34.0 & 35.5 & 0.85 \\
LAS & 25.9 & 28.5 & 0.13 \\
LAI & 23.6 & 26.7 & $\mathbf{0 . 0 0}$ \\
\hline
\end{tabular}

U de Mann-Whitnev / $\mathrm{b}<0.05$ 


\section{Comparación de dimensiones oclusales (procedencia/ 4 años de edad)}

En la tabla 4, se obtuvieron los valores promedios de las dimensiones oclusales para niños de 4 años.

Para Lima fueron:

- Ancho bicanino superior $29.0 \mathrm{~mm}$, ancho bicanino inferior $23.4 \mathrm{~mm}$.

- Ancho bimolar superior $39.2 \mathrm{~mm}$, ancho bimolar inferior $33.0 \mathrm{~mm}$.

- Longitud de arco superior $26.8 \mathrm{~mm}$ y longitud de arco inferior $23.5 \mathrm{~mm}$.

Para Yamango fueron:

- Ancho bicanino superior $31.2 \mathrm{~mm}$, ancho bicanino inferior $25.5 \mathrm{~mm}$.

- Ancho bimolar superior $41.4 \mathrm{~mm}$, ancho bimolar inferior $35.1 \mathrm{~mm}$.

- Longitud de arco superior $28.7 \mathrm{~mm}$ y longitud de arco inferior $25.4 \mathrm{~mm}$.

Al comparar los valores de las dimensiones oclusales, se obtuvo una diferencia estadística altamente significativa $(\mathrm{p}<0.01)$, para el ancho bicanino superior, longitud de arco superior y longitud de arco inferior, y una diferencia esta-

Tabla 4. Comparación de dimensiones o clusales (procedencia/ 4 años de edad).

\begin{tabular}{lccc}
\hline & \multicolumn{3}{c}{ 4 años } \\
& Yamango & Lima & P \\
\hline ABCS & 29.0 & 31.2 & $\mathbf{0 . 0 0}$ \\
ABCI & 23.4 & 25.5 & $\mathbf{0 . 0 1}$ \\
ABMS & 39.2 & 41.4 & 0.13 \\
ABMI & 33.0 & 35.1 & $\mathbf{0 . 0 4}$ \\
LAS & 26.8 & 28.7 & $\mathbf{0 . 0 0}$ \\
LAI & 23.5 & 25.4 & $\mathbf{0 . 0 0}$ \\
\hline
\end{tabular}

U de Mann-Whitney / $p<0.05$ dística significativa en el ancho bicanino inferior $y$ ancho bimolar inferior $(p<0.05)$.

\section{Comparación de dimensiones oclusales (procedencia/ 5 años de edad)}

En la tabla 5, se obtuvieron los valores promedios de las dimensiones oclusales para niños de 5 años.

Para Lima fueron:

- Ancho bicanino superior $29.2 \mathrm{~mm}$, ancho bicanino inferior $23.6 \mathrm{~mm}$.

- Ancho bimolar superior $40.0 \mathrm{~mm}$, ancho bimolar inferior $35.1 \mathrm{~mm}$.

- Longitud de arco superior $26.1 \mathrm{~mm}$ y longitud de arco inferior $23.2 \mathrm{~mm}$.

Para Yamango fueron:

- Ancho bicanino superior $31.2 \mathrm{~mm}$, ancho bicanino inferior $24.9 \mathrm{~mm}$.

- Ancho bimolar superior $40.8 \mathrm{~mm}$, ancho bimolar inferior $35.6 \mathrm{~mm}$.

- Longitud de arco superior $28.4 \mathrm{~mm}$ y longitud de arco inferior $25.9 \mathrm{~mm}$.

En la comparación se obtuvo una diferencia estadística significativa $(\mathrm{p}<0.05)$ en el ancho bica-

Tabla 5. Comparación de dimensio nes o clusales (procedencia/ 5 años de edad).

\begin{tabular}{lccc}
\hline & Yamango & $\begin{array}{r}\text { 5 años } \\
\text { Lima }\end{array}$ & $\mathbf{p}$ \\
\hline ABCS & 29.2 & 31.2 & $\mathbf{0 . 0 3}$ \\
ABCI & 23.6 & 24.9 & 0.15 \\
ABMS & 40.0 & 40.8 & 0.32 \\
ABMI & 35.1 & 35.6 & 0.25 \\
LAS & 26.1 & 28.4 & $\mathbf{0 . 0 2}$ \\
LAl & 23.2 & 25.9 & $\mathbf{0 . 0 1}$ \\
\hline
\end{tabular}

U de Mann-Whitney / $p<0.05$ 
nino superior, longitud de arco superior y una diferencia altamente significativa $(\mathrm{p}<0.01)$ en la longitud de arco inferior.

\section{Discusión}

\section{Comparación de dimensiones oclusales (sexo/procedencia)}

Se obtuvieron los valores de las dimensiones oclusales de los niños en Lima y Yamango, y como se observa en la tabla 1, existe una diferencia altamente significativa en niños y niñas de Lima en el ancho bicanino inferior y ancho bimolar superior con un $\mathrm{p}=0.00$. Abu Alhaija8 encontró un $\mathrm{p}<0.05$ en el ancho bimolar superior $y$ un $\mathrm{p}<0.001$ en el ancho bimolar inferior. Por otra parte Layseca ${ }^{11}$ en su estudio, aunque no realizó comparación por sexo y mismo estado nutricional, obtuvo medidas que nos pueden servir para comparar las obtenidas en este estudio, las cuales, son para en el ancho bicanino inferior $24.06 \mathrm{~mm}$ (d.s. 0.96) y para ancho bimolar superior $41.25 \mathrm{~mm}$ (d.s. 1.30). Se puede observar que los resultados, tomando en cuenta la desviación estándar, fueron muy cercanos, incluso, habiendo diferencias en la cantidad de la muestra. Aznar ${ }^{14}$ obtuvo en la comparación por sexo diferencias altamente significativas para el ancho bicanino y bimolar con un $\mathrm{p}=0.00$

En la comparación por sexo en Yamango se observó una diferencia estadística en el ancho bicanino superior con $29.8 \mathrm{~mm}$ para niños y 28.8 $\mathrm{mm}$ para niñas con un $\mathrm{p}=0.05$. Moreno ${ }^{10}$ obtuvo la misma diferencia estadística $(p<0.05)$ en niños con estado nutricional normal, lo curioso de los resultados obtenidos en su estudio, fue que si bien se coincide con una diferencia estadística en la misma dimensión oclusal, el promedio del valor difiere. Al comparar los resultados de
Yamango obtenidos en este estudio con los resultados de Moreno ${ }^{10}$ de niños con desnutrición crónica, determinamos que son más parecidos. Esta similitud se debería a que Yamango y Talara están ubicadas en el Departamento de Piura. Por último, el dimorfismo sexual también influye en el aspecto antropométrico, ya que los niños tienen las medias aritméticas mayores que las niñas, como lo confirmo Tsai ${ }^{15}$ en su estudio; en este estudio las diferencias estadísticas entre ellos no fueron tan marcadas como se esperaba.

\section{Comparación de dimensiones oclusales (procedencia/sexo)}

En la comparación por procedencia según sexo (tabla 2), en hombres de Lima se observa una diferencia significativa en la longitud de arco superior con un $\mathrm{p}=0.05$; en longitud de arco inferior un diferencia altamente significativa con un $p=0.00$. En el caso de las mujeres también se encontraron diferencias significativas en el ancho bicanino superior, en el ancho bicanino inferior, en el ancho bimolar superior, longitud de arco superior y en la longitud de arco inferior. Todas estas dimensiones obtuvieron un $\mathrm{p}=0.00$, excepto el ancho bimolar superior con un $\mathrm{p}=0.01$. Tsai ${ }^{15}$ en su estudio, confirmó que las giroversiones de los dientes deciduos producen alteración en las medidas de las dimensiones oclusales; y Camporessi ${ }^{16}$, sostuvo que la dimensión que ha sufrido mayor variación debido al paso del tiempo en los últimos 50 años fue la longitud de arco, dándole la causa principal a cambios en hábitos alimenticios y aumento de patologías respiratorias, causando un futuro apiñamiento en la dentición permanente.

En la tabla 2, se encontró mayor diferencia en mujeres que en hombres. Estas diferencias se pueden deber primero al tipo de alimentación 
que se tiene en distintos lugares, Camporesi ${ }^{16}$ en su estudio al comparar dos muestras de los años 1950 y 1990, lo adjudicó como un factor en las maloclusiones, los alimentos procesados serían la principal causa de impedir un adecuado desarrollo maxilar, pero se contradice con lo encontrado en este estudio, ya que los niños más propensos a ingerir alimentos procesados son los de Lima, y éstos tienen mayores medidas al compararlos con los niños de Yamango. También el factor ambiental (hipoxia) podría jugar un papel muy importante que no puede ser percibido. Según Gonzáles ${ }^{17}$ los niños de altura antropométricamente son menores a los del nivel del mar, pero estas medidas se van compensando a medida que van llegando a la edad adulta. Un dato importante de su estudio fue que el sexo femenino tiene mayor capacidad de resistencia frente a factores adversos, tales como la malnutrición, enfermedad o la vida en grandes alturas.

Comparando estos resultados con los de More$\mathrm{no}^{10}$, son diferentes, esto se puede deber a la variación con respecto al rango de edad que utilizó (de 4 a 8 años), aunque se separó la muestra en dos grupos, dentición decidua y mixta, no se especificó la cantidad de cada una. Los resultados obtenidos de Yamango se asemejan más a los obtenidos por Layseca ${ }^{11}$ en niños con desnutrición crónica que en niños con nutrición normal. Con respecto al estudio de Williams ${ }^{9}$ también difieren los resultados, siendo el más resaltante en el ancho bicanino inferior con $20.8 \mathrm{~mm}$ respecto a este estudio que se obtuvo un $25.1 \mathrm{~mm}$.

\section{Comparación de dimensiones oclusales (procedencia/ 3 años de edad)}

Los resultados obtenidos en la tabla 3, comparación por procedencia según la edad a los 3 años, se obtiene una diferencia estadística $(p<0.05)$ importante en la longitud de arco inferior $(\mathrm{p}=0.00)$, Se tiene que tener cuidado al interpretar esta medida debido al tamaño del grupo de 3 años tanto de Lima y Yamango por ser pequeño. Aznar ${ }^{14}$ obtuvo tanto para el ancho bicanino superior $(27.51 \mathrm{~mm})$ e inferior $(22.87 \mathrm{~mm})$ como para el ancho bimolar superior $(40.41 \mathrm{~mm})$ e inferior $(35.11 \mathrm{~mm})$, medidas relativamente semejantes a las obtenidas en este estudio.

\section{Comparación de dimensiones oclusales (procedencia/ 4 años de edad)}

Los resultados obtenidos en la tabla 4, comparación por procedencia según la edad a los 4 años, se obtienen cifras interesantes, como diferencias significativas $(p<0.05)$ en ancho bicanino superior, longitud de arco superior y longitud de arco inferior con un valor $p=0.00$. En el ancho bicanino inferior se obtuvo un valor de $\mathrm{p}=0.01$ y el ancho bimolar inferior se obtuvo un valor de $p=0.04$. Aznar ${ }^{14}$ obtuvo tanto para el ancho bicanino superior $27.29 \mathrm{~mm}$ e inferior $22.41 \mathrm{~mm}$, como para el ancho bimolar superior $40.11 \mathrm{~mm}$ e inferior $35.58 \mathrm{~mm}$.

\section{Comparación de dimensiones oclusales (procedencia/ 5 años de edad)}

En la tabla de comparación de procedencia por edad de 5 años, se obtuvo una diferencia estadística $(p<0.05)$ en el ancho bicanino superior, longitud de arco superior y longitud de arco inferior. Janiszewska ${ }^{12}$ en su estudio con niños de 5 años, también encontró una diferencia estadística en el ancho bicanino superior con un $\mathrm{p}=0,05$.

Los resultados de Moorrees ${ }^{18,19}$, tanto para el ancho bicanino superior $(28.8 \mathrm{~mm})$ y para el ancho bicanino inferior $(22.3 \mathrm{~mm})$, se asemejan a 
Tabla 6. Comparación de resultados con otros estudios en el Perú.

\begin{tabular}{lccccccc}
\hline & \multicolumn{2}{c}{ Lima } & \multicolumn{2}{c}{ Yamango } & $\begin{array}{c}\text { Moreno } \\
\text { (Piura) }\end{array}$ & $\begin{array}{c}\text { Williams } \\
\text { (Lima) }\end{array}$ & $\begin{array}{c}\text { Layseca } \\
\text { (Cuzco) }\end{array}$ \\
\hline XBCS & $\mathbf{3 1 . 1}$ & $\mathbf{1 . 8 3}$ & $\mathbf{2 9 . 2}$ & $\mathbf{2 . 2 2}$ & 31.8 & 27.5 & 31.1 \\
ABCI & $\mathbf{2 5 . 1}$ & $\mathbf{2 . 5 8}$ & $\mathbf{2 3 . 6}$ & $\mathbf{2 . 1 8}$ & 25.5 & 20.8 & 24.7 \\
ABMS & $\mathbf{4 0 . 9}$ & $\mathbf{2 . 0 6}$ & $\mathbf{3 9 . 6}$ & $\mathbf{2 . 3 2}$ & 44.6 & 40.1 & 41.7 \\
ABMI & $\mathbf{3 5 . 3}$ & $\mathbf{2 . 5 7}$ & $\mathbf{3 4 . 1}$ & $\mathbf{3 . 2 1}$ & 35.3 & 35.4 & 36.4 \\
LAS & $\mathbf{2 8 . 5}$ & $\mathbf{2 . 1 9}$ & $\mathbf{2 6 . 3}$ & $\mathbf{2 . 0 6}$ & 24.7 & 27.6 & 26.9 \\
LAI & $\mathbf{2 5 . 7}$ & $\mathbf{2 . 3 6}$ & $\mathbf{2 3 . 4}$ & $\mathbf{1 . 9 3}$ & 21.9 & 24.3 & 24.3 \\
\hline
\end{tabular}

Medida Estadística: Media Aritmética

los obtenidos en este estudio para los niños de Yamango; mientras que la longitud de arco superior $(29.2 \mathrm{~mm})$ y la longitud de arco inferior $(25.8 \mathrm{~mm})$ se asemejan a los obtenidos para niños de Lima. Aznar ${ }^{14}$ encontró resultados parecidos para el ancho bicanino superior (27.52 $\mathrm{mm}$ ), Thilander ${ }^{20}$ obtuvo para la longitud de arco superior $23.25 \mathrm{~mm}$ (D.S 1.70) y longitud de arco inferior $20.5 \mathrm{~mm}$ (D.S. 1.27).

Si realizamos una comparación por edades con respecto a los promedios de las dimensiones oclusales, podremos notar que a medida que va aumentado la edad, los valores son menores, lo que difiere de lo expuesto por Canut ${ }^{21}$ y Bishara $^{22}$, el primero menciona que dentro de los 2 primeros años de vida hasta los 18 años existe un aumento de diámetro intercanino e intermolar, el cual no se observa en este caso, el segundo menciona que entre los 3 a 5 años de edad existe un aumento del ancho intermolar superior de 2 $\mathrm{mm}$ e inferior de $1.5 \mathrm{~mm}$, que tampoco se observa. En la longitud de arco superior e inferior de los niños de 4 para 5 años, encontraremos una ligera disminución de la medida. En el caso de Yamango se encontró que para la longitud de arco superior, el promedio disminuye de 26.8 $\mathrm{mm}$ a $26.1 \mathrm{~mm}(-0.7 \mathrm{~mm})$, y para la longitud de arco inferior el valor disminuye de $23.5 \mathrm{~mm}$ a $23.2 \mathrm{~mm}(-0.3 \mathrm{~mm})$ y en el caso de Lima se evidenció una disminución para la longitud de arco superior de $28.7 \mathrm{~mm}$ a $28.4 \mathrm{~mm}(-0.3 \mathrm{~mm})$ y para la longitud de arco inferior aumenta de $25.4 \mathrm{~mm}$ a $25.9 \mathrm{~mm}(+0.05 \mathrm{~mm})$, este último resultado difiere de los demás, aunque si es normal un ligero desplazamiento de las molares deciduas hacia mesial debido a la fuerza de erupción de la primera molar permanente, esta medida se ve incrementada. Se tiene que tener cuidado con estos resultados debido a baja cantidad y paridad de las muestras clasificadas por edad.

\section{Comparaciones adicionales}

\section{Comparación de resultados con otros estudios en el Perú}

En la tabla 6 se observan los distintos datos de estudios más recientes realizados en Perú, los resultados obtenidos difieren unos de otros, en el caso de Moreno ${ }^{10}$ (Talara - Piura) y Layseca ${ }^{11}$ 
(Saylla-Cusco) que obtuvieron resultados para niños con estado nutricional normal y desnutrición crónica, estos últimos o se consideran en la tabla, pero se tuvieron en cuenta para algunas comparaciones anteriores. Para obtener una respuesta a la diferencia de datos, se tiene que tener en cuenta la cantidad de muestra de cada estudio y la etnia de los sujetos estudiados (Moyers $^{23}$ encontró que en negros con mismo nivel socioeconómico que blancos en Estados Unidos, están más adelantados en las medidas de crecimiento durante el periodo de los dientes primarios).

Si bien el Perú está en su mayoría compuesto por raza mestiza, observamos variación; el lugar donde se realizaron las investigaciones (la altitud presente como un factor ambiental), el nivel socioeconómico, el estado nutricional y la técnica utilizada también influye con respecto a los resultados.

\section{Comparación de otros resultados con otros estudios}

En la tabla 7, se observan los resultados de esta investigación con los de otros autores realizadas en España, Suecia, Polonia y Jordania. Lo sorprendente es que las medidas obtenidas son bastantes similares, todo a lo contrario que se pensaba obtener debido a la diferencia racial, ambiental y socioeconómica.

\section{Conclusiones}

Las conclusiones del estudio fueron las siguientes:

En el presente estudio, se determinaron los valores promedio y desviación estándar para las muestras de niños de 3 a 5 años en dentición decidua completa atendidos en la Clínica Estomatológica Central de la UPCH y Yamango en el Departamento Piura (ver tablas).

Las dimensiones oclusales de los hombres atendidos en la Clínica Estomatológicas Central de la UPCH comparadas con las dimensiones oclusales de los hombres de Yamango fueron significativamente mayores en el ABCI y ABMS; y las dimensiones oclusales de las mujeres atendidas en la Clínica Estomatológica Central de la UPCH comparadas con las dimensiones oclusales de las mujeres de Yamango, fueron significativamente mayores excepto en el ABCS.

Las dimensiones oclusales de los hombres atendidos en la Clínica Estomatológicas Central de

Tabla 7. Comparación de resultados con otros estudios.

\begin{tabular}{lcccccccc}
\hline & \multicolumn{2}{c}{ Lima } & \multicolumn{2}{c}{ Yamango } & $\begin{array}{c}\text { Aznar } \\
\text { (España) }\end{array}$ & $\begin{array}{c}\text { Thilander } \\
\text { (Suecia) }\end{array}$ & $\begin{array}{c}\text { Janiszewska } \\
\text { (Polonia) }\end{array}$ & $\begin{array}{c}\text { Abu Alhaija } \\
\text { (Jordania) }\end{array}$ \\
\hline & X & D.S. & X & D.S. & & & & \\
ABCS & $\mathbf{3 1 . 1}$ & $\mathbf{1 . 8 3}$ & $\mathbf{2 9 . 2}$ & $\mathbf{2 . 2 2}$ & 27.53 & 29.45 & 29.4 & 29.58 \\
ABCI & $\mathbf{2 5 . 1}$ & $\mathbf{2 . 5 8}$ & $\mathbf{2 3 . 6}$ & $\mathbf{2 . 1 8}$ & 22.73 & 22.95 & 23.4 & 23.5 \\
ABMS & $\mathbf{4 0 . 9}$ & $\mathbf{2 . 0 6}$ & $\mathbf{3 9 . 6}$ & $\mathbf{2 . 3 2}$ & 40.46 & 38.25 & 39.5 & 39.13 \\
ABMI & $\mathbf{3 5 . 3}$ & $\mathbf{2 . 5 7}$ & $\mathbf{3 4 . 1}$ & $\mathbf{3 . 2 1}$ & 35.83 & 34.15 & 35.4 & 34.83 \\
LAS & $\mathbf{2 8 . 5}$ & $\mathbf{2 . 1 9}$ & $\mathbf{2 6 . 3}$ & $\mathbf{2 . 0 6}$ & - & 23.25 & 26.6 & 25.12 \\
LAI & $\mathbf{2 5 . 7}$ & $\mathbf{2 . 3 6}$ & $\mathbf{2 3 . 4}$ & $\mathbf{1 . 9 3}$ & - & 20.5 & 23.6 & 22.67 \\
\hline
\end{tabular}

Medida Estadística: Media Aritmética 
la UPCH comparadas con las dimensiones oclusales de los hombres de Yamango fueron significativamente mayores en LAS y LAI; y las dimensiones oclusales de las mujeres atendidas en la Clínica Estomatológica Central de la UPCH con las dimensiones oclusales de las mujeres de Yamango, fueron significativamente mayores, excepto en el ABMI.
Las dimensiones oclusales de los niños atendidos en la Clínica Estomatológicas Central de la UPCH comparadas con las dimensiones oclusales de los niños de Yamango a los 3 años, fueron significativamente mayores en LAI; a los 4 años fueron significativamente mayores, excepto en ABMS; y a los 5 años fueron significativamente mayores en ABCS, LAS y LAI.

\section{Referencias}

1. Baume L. Physiological tooth migration and its significance for the development of occlusion. II. The biogenesis of accessional dentition. J Dent Res 1950; 29: 331-37.

2. Moorrees C, Gron A, LebrelR, Yen PandFolickF, Growth Study of the dentition. Arevieew. Am JOrthond 1969;44:600-615. Moyers R, Van der linden F, Riolo M, McNamara J. Standard for human oclussal development. Monograph 5.1976. Craniofacial Growth Series. Center For Human Growth and Development. University of Michigan. An Arbor.

3. Lux C., Conradt C., Burden D., Komposch G. Transverse development of the craniofacial skeleton and dentition between 7 and 15 years of age-a longitudinal postero-anterior cephalometric study. The European Journal of Orthodontics 2004 26(1):31-42.

4. Aliaga A. Dimensiones transversales esqueléticas y del arco maxilar en pacientes con secuela de fisura labio alveolo palatina unilateral. [tesis de bachiller] Lima (Perú). Facultad de Odontología. UNMSM; 2010.

5. Luz D`Escriván De Saturno y col. Ortodoncia en Dentición Mixta. Editorial Amolca, Colombia 2007.

6. Hixon E, Oldfather R. Estimation of the size of Unerupted Cuspid and Bicuspid Teeth. Angle Orthodontist 1958, Oct. 28(4): $236-40$.

7. Alhaija A, Qudeimat A. Occlusion and tooth/ arch dimensions in the primary dentition of preschool Jordanian Children. Division of Orthodontics and Division of Pediatric dentistry, Jordan University of Science and Technology, Irbid, Jordan. Int J Ped Dent 2003; 13: 230-239.

8. Williams F, Valverde R, Meneses A. Dimensiones de arcos y relaciones oclusales en dentición decidua completa. Rev. Estomatol Herediana 2004; 14(1-2): 22-16.

9. Moreno K, Meneses A, Morzán E, Dimensiones de arcos dentarios en niños de 4 a 8 años de edad con diferente estado nutricional. Talara-Piura, Rev Estomatol Herediana 2004; 14 (1-2): 18 - 21.

10. Layseca L, Soto K, Cosio H. Estudio Comparativo de dimensiones de arcos dentarios en niños desnutridos crónicos y eutróficos con dentición decidua y mixta primera fase- Instituciones Educativas de Saylla- Cusco, 2006. bvrevistas unmsm 2006 SITUA -15 (1,2).

11. Janiszewska-Olszowska. Spacing in deciduous dentition of Polish children in relation to tooth size and dental arch dimensions. Arch Oral Biol 2009 May 54(5): 397-402. Epub 2009 Feb 23.

12. Nanda R y col. Age changes in the occlusal pattern in deciduous dentition. J dent Res 1973; 52 (2):221-4.

13. Aznar T, Galan F, Marín I, Domínguez A. Dental Arch Diameters and Relationships to Oral Habits. Angle Orthodontist 2006, Vol 76, No 3.

14. Tsai H. Tooth-position, arch-size and arch-shape in the primary dentition. ASDJ Dent Child 2001 Jan-Feb: 68(1):17-22.

15. Camporesi M, Marinelli A, Dental Arch dimension and tooth wear in two samples of children in the 1950s and 1990s. BDJ 2009; 207(12):E24.

16. Gonzáles G. Hipoxia: Investigaciones Básicas y Clínicas: Homenaje a Carlos Monge Cassinelli. Universidad Peruana Cayetano Heredia. Facultad de Medicina. 1993 Pág. 321-337 
17. Moorrees, Reed R. Changes in Dental Arch Dimensions Expressed on the Basis of Tooth Eruption as a Measure of Biologic Age. J Dent Res. 1965 Jan-Feb;44(1):129-41.

18. Moorrees. Normal Variation in Dental Development Determined with Reference t Tooth Eruption Status. J Dent Res.1965 Jan- Feb;44(1):161-173.

19. Thilander B. Dentoalveolar development in subjects with normal occlusion a Longitudinal study between the ages of 5 and 31 years. Eur J Orthod 2009 Apr; 31(2): 109-20.

20. Canut J. Ortodoncia Clínica. Salvat Editores S.A., Barcelona - España 1991.

21. Bishara SE. Ortodoncia .Editorial: McGraw Hill Interamericana Editores, México 2003.

22. Moyers RE. Manual de Ortodoncia 4ta Edición. Editorial Médica Panamericana, Buenos Aires - Argentina 1992.

Texto traducido por: Antonio J. Nogueira

Recibido: 15-02-2012

Aceptado: 18-03-2012

Correspondencia: ruben_zambrano29@hotmail.com 